\title{
Identification of Candida glabrata Transcriptional Regulators that Govern Stress Resistance and Virulence
}

\author{
Elan E. Filler ${ }^{1}$, Yaoping Liu ${ }^{1}$, Norma V. Solis ${ }^{1}$, Luis F. Diaz ${ }^{2}$, John E. Edwards, Jr. ${ }^{1,3}$, Scott G. \\ Filler $^{1,3 *}$, Michael R. Yeaman ${ }^{2,3 *}$ \\ ${ }^{1}$ Division of Infectious Diseases, Lundquist Institute for Biomedical Innovation at Harbor-UCLA \\ Medical Center, Torrance, CA \\ ${ }^{2}$ Division of Molecular Medicine, Lundquist Institute for Biomedical Innovation at Harbor- \\ UCLA Medical Center, Torrance, CA \\ ${ }^{3}$ David Geffen School of Medicine at UCLA, Los Angeles, CA
}

Running Title: C. glabrata Transcriptional Regulators

*Corresponding authors: sfiller@ucla.edu mryeaman@ucla.edu 


\begin{abstract}
The mechanisms by which Candida glabrata resists host defense peptides and caspofungin are incompletely understood. To identify transcriptional regulators that enable C. glabrata to withstand these classes of stressors, a library of 215 C. glabrata transcriptional regulatory deletion mutants was screened for susceptibility to both protamine and caspofungin. We identified 8 mutants that had increased susceptibility to both host defense peptides and caspofungin. Of these mutants, 6 were deleted for genes that were predicted to specify proteins involved in histone modification. These genes were ADA2, GCN5, SPT8, HOS2, RPD3, and SPP1. The ada2 $\Delta$ and $g c n 5 \Delta$ mutants also had increased susceptibility to other stressors such as $\mathrm{H}_{2} \mathrm{O}_{2}$ and SDS. In the Galleria mellonella model of disseminated infection, the ada2 $\Delta$ and gcn $5 \Delta$ mutants had attenuated virulence, whereas in neutropenic mice, the virulence of the ada2 $\Delta$ and $r p d 3 \Delta$ mutants was decreased. Thus, histone modification plays a central role in enabling $C$. glabrata to withstand host defense peptides and caspofungin, and Ada2 is essential for the maximal virulence of this organism during disseminated infection.
\end{abstract}




\section{Introduction}

In the United States of America, Candida glabrata causes approximately one third of cases of hematogenously disseminated candidiasis $(1,2)$. The echinocandin antifungal agents are currently the primary treatment for disseminated candidal infections, especially those caused by $C$. glabrata (3). Of significant concern, echinocandin antifungal drugs are being isolated with increasing frequency (4). In some medical centers, up to $25 \%$ of $C$. glabrata isolates are either intermediately susceptible or resistant to echinocandins (5) Many strains of C. glabrata are resistant to echinocandins because of mutations in FKS1 or FKS2 that encode 1,3 $\beta$-glucan synthases, the target enzymes of these drugs (6). However, other strains are resistant to echinocandins by other, unknown mechanisms $(2,7)$.

Host defense peptides play key roles in the host defense against $C$. glabrata infections. These peptides, such as $\alpha$-defensins, $\beta$-defensins, cathelicidins and LL-37, are produced by leukocytes and the epithelial cells that line cutaneous and mucosal barriers $(8,9)$. For $C$. glabrata to colonize the host and persist within the deep tissues, it must be able to resist inhibition or killing by these host defense peptides. While some strains of C. glabrata are readily killed by $\beta$-defensins, other strains are relatively resistant (10). The mechanisms by which C. glabrata resists host defense peptides are not fully understood.

To gain a deeper understanding of the mechanisms by which $C$. glabrata resists both echinocandins and host defense peptides, we screened a library of 215 C. glabrata transcriptional regulator mutants for strains that were susceptible to the echinocandin, caspofungin and the host defense peptide, protamine. Remarkably, of the 8 strains that were found to susceptible to both compounds, 6 contained mutations in genes whose products are predicted to function in histone modification including acetylation, deacetylation, and methylation. Among these mutants, the 
$\triangle a d a 2$ and $\Delta g c n 5$ mutants were also found to have increased sensitivity to hydrogen peroxide and SDS. Both the $\triangle a d a 2$ and the $\Delta r p d 3$ mutants had attenuated virulence during disseminated infection in neutropenic mice. These results indicate that in C. glabrata, histone modification is required for the organism to resist caspofungin and protamine, and that Ada2 and Rbd3 govern virulence during disseminated infection.

\section{Results}

\section{Screening the transcription factor deletion mutant library identified eight genes that} governed susceptibility to protamine and caspofungin. In total, 215 C. glabrata deletion mutants were screened for susceptibility to protamine and caspofungin. This screen identified 17 mutants that had increased susceptibility to protamine alone and 8 that had increased susceptibility to caspofungin alone, relative to the wild-type strain (Supplemental Table 1). Eight mutants had increased susceptibility to both protamine and caspofungin (Table 1; Fig. 1). Of the genes that were mutated in these eight strains, six specified proteins involved in histone modification. These proteins included Ada2, Gcn5, and Spt8, which are components of the SAGA histone acetyltransferase complex (11), Hos2 and Rpd3, which are members of same family of histone deacetylases (12), and Spp1, a component of the COMPASS histone methyltransferase complex (13). To verify that Ada2, Gcn5, Hos2, Rpd3, and Spp1 governed susceptibility to protamine and caspofungin, we complemented each of the $C$. glabrata mutants with an intact allele of the gene that was disrupted. Complementation restored the wild-type phenotype in all strains (Figure 1). These results indicate that histone modification is the key mechanism by which $C$. glabrata resists host defense peptides and caspofungin.

Two additional mutants, $c r z 1 \Delta$ and $m k s 1 \Delta$, were identified that had increased susceptibility to both protamine and caspofungin. Neither Crz1 nor Mks1 are involved in histone 
modification (Table 1). Crz1 is a transcription factor in the calcineurin signaling pathway (14), and Mks1 is a negative transcriptional regulator that inhibits multiple signaling pathways such as the target of rapamycin (TOR) and Ras-cyclic AMP pathways $(15,16)$. These data demonstrate that diverse signaling pathways govern susceptibility to protamine and caspofungin.

Ada2 and Gcn5 govern resistance to multiple stressors. Next, we evaluated whether histone modification is required for C. glabrata to withstand stressors other than protamine and caspofungin. We determined that the ada2 $\Delta$ mutant had increased susceptibility to calcofluor white, $\mathrm{H}_{2} \mathrm{O}_{2}$, SDS and $\mathrm{NaCl}$ relative to the wild-type and ada2 $\Delta+A D A 2$ complemented strains (Fig. 2). The gcn5 $\Delta$ mutant had increased susceptibility to $\mathrm{H}_{2} \mathrm{O}_{2}$ and $\mathrm{SDS}$ as compared to the wild-type and $g c n 5 \Delta+G C N 5$ complemented strains. By contrast, the $\operatorname{rpd} 3 \Delta, \operatorname{hos} 2 \Delta$, and $\operatorname{spp} 1 \Delta$ mutants had wild-type susceptibility to all stressors tested. Thus, the members of the SAGA complex, particularly Ada2, are required for C. glabrata to withstand multiple different types of stress.

Protamine was used as a representative host defense peptide in the screening assays. To verify that Ada2 governed the susceptibility of $C$. glabrata to human host defense peptides, we tested the susceptibility of the ada2 $\Delta$ mutant to human neutrophil defensin-1 (hNP-1) and human $\beta$-defensin-2 (hBD-2) using a radial diffusion assay. As compared to the wild-type strain and the ada2 $\Delta+A D A 2$ complemented strains, the ada2 $\Delta$ mutant had increased susceptibility to both of these host defense peptides (Fig. 3).

Ada2 is required for virulence. We hypothesized that mutants that were susceptible to both protamine and caspofungin would also have attenuated virulence. To test this hypothesis, we tested the virulence $a d a 2 \Delta, \operatorname{gcn} 5 \Delta, \operatorname{rpd} 3 \Delta, \operatorname{hos} 2 \Delta$, and $\operatorname{spp} 1 \Delta$ mutants in two models of disseminated candidiasis, G. mellonella larvae and immunosuppressed mice. In the G. mellonella 
model, each mutant was tested at inocula of $2.5 \times 10^{6}$ and $5 \times 10^{6}$ organisms per larvae. Of the five mutants tested, only the ada2 $\Delta$ and gcn5 $\Delta$ mutants had virulence defects. Larvae infected with either the high or low inoculum of the $\Delta a d a 2$ mutant had significantly longer survival than those infected with the wild-type strain and the ada2 $\Delta+$ ADA2 complemented strain (Fig 4). Larvae infected with the low inoculum of the gcn5 $\Delta$ mutant also survived significantly longer than larvae infected with the controls strain. However, when injected at the high inoculum, the virulence defect of the gcn5 $\Delta$ mutant was no longer apparent. Collectively, these results demonstrate that Ada2, and to a lesser extent Gcn5, are required for the full virulence of $C$. glabrata in G. mellonella.

The virulence of the five transcriptional regulatory mutants was also tested in the neutropenic mouse model of disseminated candidiasis using kidney fungal burden as the primary endpoint. Both the ada2 $\Delta$ and $r p d 3 \Delta$ mutants had significant virulence defects (Fig. 5). The median kidney fungal burdens of mice infected with these mutants was 50-fold and 80-fold lower than that of mice infected with the wild-type strain. In contrast to its attenuated virulence in G. mellonella, the gcn5 mutant had wild-type virulence in mice.

\section{Discussion}

The results obtained from screening a library of $C$. glabrata deletion mutants revealed that histone modification plays a central role in governing the susceptibility of the organism to protamine and caspofungin. Of the eight transcriptional regulators that identified in this screen, six were predicted to be components of major regulatory complexes that modify histones. Furthermore, Ada2, an adapter protein that is a critical component of multiple histone 
acetyltransferase complexes, and RPD3, a histone deacetylase, are required for the maximal virulence of $C$. glabrata in neutropenic mice.

Ada2, Gcn5, and Spt8 were found to be necessary for C. glabrata to withstand both protamine and caspofungin. These proteins are members of the SAGA histone acetylase complex $(11,17)$. Ada2 functions as an adapter protein, Gcn5 is an acetyltransferase, and Spt8 is a regulatory protein that is required for the function of the SAGA complex (11). Ada2 and Gcn5 are also members of multiple other histone acetyltransferase complexes $(18,19)$. Although the phenotypes of the $a d a 2 \Delta$ and $g c n 5 \Delta$ mutants were similar, the ada2 $\Delta$ mutant had increased susceptibility to a broader range of stressors than the gcn5 $\Delta$ mutant. For example, the ada2 $\Delta$ mutant had increased susceptibility to calcofluor white and $\mathrm{NaCl}$, whereas the $g \operatorname{cn} 5 \Delta$ mutant did not. Also, while the ada2 $\Delta$ mutant had a virulence defect in both $G$. mellonella and mice, the gcn5 $\Delta$ mutant had only a modest virulence defect in $G$. mellonella and had wild-type virulence in mice. In Candida albicans, deletion of both $A D A 2$ and GCN5 results in attenuated virulence $(20,21)$. Our results indicate that $C$. glabrata differs from $C$. albicans in that Ada2 plays a greater role than Gcn5 in stress resistance and virulence.

The finding that Ada2 is particularly important for the $C$. glabrata to tolerate a wide variety of stressors provides a compelling explanation as to why this mutant had reduced virulence in both $G$. mellonella and mice. Two other groups have also reported that $C$. glabrata ada2 $\Delta$ mutants have increased susceptibility to a broad range of stressors $(22,23)$. In agreement with our results, Kounatidis et al. found that an ada2 $\Delta$ mutant had attenuated virulence in a Drosophila model of gastrointestinal infection (22). By contrast, Yu et al. found that an ada2s mutant actually had increased virulence in mice, as manifested by enhanced lethality and increased organ fungal burden (23). The reason for why the virulence data of Yue et al. differed 
from ours is unclear, but may be due to differences in the background strain of C. glabrata and the immunosuppression regimen that were used.

We found that both the $\operatorname{rpd} 3 \Delta$ and hos $2 \Delta$ mutants has increased susceptibility to protamine and caspofungin, and that the $r p d 3 \Delta$ mutant had attenuated virulence in mice. In agreement with our results, Schwarzmuller et al. also determined that a C. glabrata rpd3A mutant had increased susceptibility to caspofungin (24). Rpd3 and Hos2 are members of the same class of histone deacetylases, and in $S$. cerevisiae, $r p d 3 \Delta$ and hos $2 \Delta$ mutants have overlapping phenotypes (12). Other members of this class of histone deacetylases include Hda1, Hos1, and Hos3. We found that the hdal $\Delta$ mutant had increased resistance to protamine and wild-type susceptibility to caspofungin, and that the hos $1 \Delta$ and hos $3 \Delta$ mutants had wild-type susceptibility to both protamine and caspofungin. Thus, although these histone deacetylases are members of the same family, they have different functions in C. glabrata.

It was found that the $\operatorname{spp} 1 \Delta$ mutant had increased susceptibility to protamine and caspofungin. Spp1 is predicted to be a member of the COMPASS complex. In S. cerevisiae, this complex functions as a histone methyltransferase, adding three methyl groups to lysine 4 of histone H3 by the Set1 methyltransferase. Spp1 stabilizes Set1 and retards its degradation (26). Our finding data suggest that the COMPASS complex is required for C. glabrata to withstand to protamine and caspofungin these stressors. The phenotype of a $C$. glabrata sppl $1 \Delta$ mutant has not been reported previously and a $\Delta$ set 1 mutant was not present in the library. However, it is known that a $C$. albicans set $1 \Delta / \Delta$ mutant is hyperfilamentous and has attenuated virulence in mice (27). Our experimental results predict that the $\Delta$ set 1 mutant would be likely susceptible to both protamine and caspofungin, and perhaps have attenuated virulence. 
Testing the virulence of the various $C$. glabrata mutants in both G. mellonella and mice enabled us to determine the extent of concordance between these two models of disseminated candidiasis. The ada2 $\Delta$ mutant had attenuated virulence in both models, and the hos $2 \Delta$ and spp $1 \Delta$ mutants had wild-type virulence in both models. By contrast, the gcn5 $\Delta$ mutant had reduced virulence only in $G$. mellonella, and the $r p d 3 \Delta$ mutant had reduced virulence only in mice. These results suggest that although the G. mellonella model has modest predictive value, any $C$. glabrata mutant that has reduced virulence in this model should be tested in mice to verify the results.

Collectively, the data presented herein suggest that in C. glabrata, histone modification is a central mechanism by which the organism withstands stress due to host defense peptides and echinocandin antifungal drugs. Ada2 is also necessary for maximal virulence of this organism during disseminated infection. Although the transcriptional adapter- $2 \alpha$ is the human counterpart of C. glabrata Ada2, these two proteins share only $29 \%$ amino acid identity. Thus, Ada2 holds promise as a potential antifungal drug target.

\section{Materials and Methods}

Deletion mutant library screening. To identify transcriptional regulators that govern resistance to protamine, a representative host defense peptide and caspofungin, a library of 215 C. glabrata transcriptional regulator deletion mutants constructed in the ATCC 2001 strain background was generously provided by Brendan Cormack at the Johns Hopkins School of Medicine (24). The susceptibility of the mutants to caspofungin and protamine was determined using an agar-dilution assay (28). The various mutants and the wild-type control strain were grown in yeast extract peptone dextrose (YPD) broth at $30^{\circ} \mathrm{C}$ in a shaking incubator overnight. 
The next day, each culture was adjusted to an $\mathrm{OD}_{600}$ of 2.7 in YPD broth. Four serial 10-fold dilutions were made in PBS, after which $3 \mu 1$ of each dilution was spotted onto YPD agar containing either $325 \mathrm{ng} / \mathrm{ml}$ of caspofungin or $3 \mathrm{mg} / \mathrm{ml}$ of protamine. After incubation at $30^{\circ} \mathrm{C}$ for $48 \mathrm{~h}$, the plates were imaged and the growth of each mutant was compared to that of the wildtype parent strain. Any mutant that differed from the wild-type strain in its susceptibility to caspofungin or protamine was independently tested at least twice to verify the results.

To assess whether selected mutants had increased susceptibility to additional stressors, the above agar dilution method was used to assess growth on calcofluor white $(400 \mu \mathrm{g} / \mathrm{ml})$, hydrogen peroxide $(15 \mathrm{mM}), \operatorname{SDS}(0.2 \%)$, and $\mathrm{NaCl}(0.55 \mathrm{M})$.

Complementation. To complement the $\Delta a d a 2, \Delta g c n 5, \Delta r p d 3, \Delta h o s 2$, and $\Delta s p p 1$ mutants, the protein coding regions plus 1000 bp of upstream and $500 \mathrm{bp}$ of downstream flanking sequences of each gene was PCR amplified from C. glabrata genomic DNA. Each resulting PCR product was cut with BamHI and XhoI and ligated into plasmid pGRB2.1+His3 (29), which had been linearized with Bam HI and XhoI. The resulting plasmids were transformed into C. glabrata using the lithium acetate method.

Radial diffusion assay. To assess the susceptibility of the wild-type, ada2 $\Delta$ muant, and ada2 $\triangle+A D A 2$ complemented strains to human host defense peptides, we employed an ultrasensitive radial diffusion assay (30). The host defense peptides used were HNP-1 and hBD-2, and they were tested at $\mathrm{pH} 7.5$.

Virulence testing. The animal studies performed for this research were approved by the Lundquist Institute Animal Care and Use Committee. The virulence of the various C. glabrata mutants was assessed in the G. mellonella larval (31) and neutropenic mouse models of disseminated infection (32). G. mellonella larvae in the final instar stage were inoculated with 
$2.5 \times 10^{6}$ or $5 \times 10^{6}$ C. albicans cells in total volume of $5 \mu$ using a Hamilton syringe. Twelve larvae were inoculated with each strain of $C$. glabrata and an additional 10 larvae were injected with $5 \mu 1$ of PBS as a negative control. The larvae were kept in a humidified incubator at $37^{\circ} \mathrm{C}$ and monitored twice daily for survival. Each strain was tested twice and the results of the two experiments were combined.

The virulence of the mutants was also tested in the neutropenic mouse model of disseminated candidiasis (32). To induce neutropenia, male Balb/c mice were administered 5fluorouracil via intraperitoneal injection at $150 \mathrm{mg} / \mathrm{kg}$ of body weight at day -1 relative to infection. The next day, they were inoculated with $10^{7} \mathrm{C}$. glabrata cells via the lateral tail vein. After 8 days of infection, the mice were euthanized, after which their kidneys were harvested, weighed, homogenized, and quantitatively cultured. Each strain was tested twice in 6 to 8 mice per experiment and the results were combined.

Statistical analysis. Using the GraphPad Prism software package, the survival of larvae infected with each mutant was compared with larvae infected with the wild-type strain and the complemented strain using the Gehan-Breslow-Wilcoxon test. The mouse organ fungal burden data were analyzed using the Kruskal Wallis test. P-values $\leq 0.05$ were considered significant.

\section{Acknowledgements}

We thank Brendan Cormack for generously supplying the transcription factor deletion library. This work was supported in part by NIH grant R01AI124566. 


\section{References}

1. Cleveland AA, Farley MM, Harrison LH, Stein B, Hollick R, Lockhart SR, Magill SS, Derado G, Park BJ, Chiller TM. 2012. Changes in incidence and antifungal drug resistance in candidemia: results from population-based laboratory surveillance in Atlanta and Baltimore, 2008-2011. Clin Infect Dis 55:1352-61.

2. Pfaller M, Neofytos D, Diekema D, Azie N, Meier-Kriesche HU, Quan SP, Horn D. 2012. Epidemiology and outcomes of candidemia in 3648 patients: data from the Prospective Antifungal Therapy (PATH Alliance(R)) registry, 2004-2008. Diagn Microbiol Infect Dis 74:323-31.

3. Pappas PG, Kauffman CA, Andes DR, Clancy CJ, Marr KA, Ostrosky-Zeichner L, Reboli AC, Schuster MG, Vazquez JA, Walsh TJ, Zaoutis TE, Sobel JD. 2016. Clinical practice guideline for the management of candidiasis: 2016 update by the Infectious Diseases Society of America. Clin Infect Dis 62:16.

4. Cleveland AA, Harrison LH, Farley MM, Hollick R, Stein B, Chiller TM, Lockhart SR, Park BJ. 2015. Declining incidence of candidemia and the shifting epidemiology of Candida resistance in two US metropolitan areas, 2008-2013: results from populationbased surveillance. PLoS ONE 10.

5. Vallabhaneni S, Cleveland AA, Farley MM, Harrison LH, Schaffner W, Beldavs ZG, Derado G, Pham CD, Lockhart SR, Smith RM. 2015. Epidemiology and risk factors for echinocandin nonsusceptible Candida glabrata bloodstream infections: data from a large multisite population-based candidemia surveillance program, 2008-2014. Open Forum Infect Dis 2.

6. Alexander BD, Johnson MD, Pfeiffer CD, Jimenez-Ortigosa C, Catania J, Booker R, Castanheira M, Messer SA, Perlin DS, Pfaller MA. 2013. Increasing echinocandin resistance in Candida glabrata: clinical failure correlates with presence of FKS mutations and elevated minimum inhibitory concentrations. Clin Infect Dis 56:1724-32.

7. Shields RK, Kline EG, Healey KR, Kordalewska M, Perlin DS, Nguyen MH, Clancy CJ. 2018. Spontaneous mutational frequency and $F K S$ mutation rates vary by echinocandin agent against Candida glabrata. Antimicrob Agents Chemother 63:01692-18.

8. Yeaman MR, Yount NY. 2003. Mechanisms of antimicrobial peptide action and resistance. Pharmacol Rev 55:27-55.

9. Yount NY, Weaver DC, Lee EY, Lee MW, Wang H, Chan LC, Wong GCL, Yeaman MR. 2019. Unifying structural signature of eukaryotic alpha-helical host defense peptides. Proc Natl Acad Sci U S A 116:6944-6953.

10. Vylkova S, Nayyar N, Li W, Edgerton M. 2007. Human beta-defensins kill Candida albicans in an energy-dependent and salt-sensitive manner without causing membrane disruption. Antimicrob Agents Chemother 51:154-61.

11. Sterner DE, Grant PA, Roberts SM, Duggan LJ, Belotserkovskaya R, Pacella LA, Winston F, Workman JL, Berger SL. 1999. Functional organization of the yeast SAGA complex: distinct components involved in structural integrity, nucleosome acetylation, and TATA-binding protein interaction. Mol Cell Biol 19:86-98.

12. Sharma VM, Tomar RS, Dempsey AE, Reese JC. 2007. Histone deacetylases RPD3 and HOS2 regulate the transcriptional activation of DNA damage-inducible genes. Mol Cell Biol 27:3199-210. 
13. Acquaviva L, Szekvolgyi L, Dichtl B, Dichtl BS, de La Roche Saint Andre C, Nicolas A, Geli V. 2013. The COMPASS subunit Spp1 links histone methylation to initiation of meiotic recombination. Science 339:215-8.

14. Miyazaki T, Yamauchi S, Inamine T, Nagayoshi Y, Saijo T, Izumikawa K, Seki M, Kakeya H, Yamamoto Y, Yanagihara K, Miyazaki Y, Kohno S. 2010. Roles of calcineurin and Crz1 in antifungal susceptibility and virulence of Candida glabrata. Antimicrob Agents Chemother 54:1639-43.

15. Dilova I, Chen CY, Powers T. 2002. Mks1 in concert with TOR signaling negatively regulates RTG target gene expression in S. cerevisiae. Curr Biol 12:389-95.

16. Matsuura A, Anraku Y. 1993. Characterization of the MKS1 gene, a new negative regulator of the Ras-cyclic AMP pathway in Saccharomyces cerevisiae. Mol Gen Genet 238:6-16.

17. Daniel JA, Grant PA. 2007. Multi-tasking on chromatin with the SAGA coactivator complexes. Mutat Res 618:135-48.

18. Eberharter A, Sterner DE, Schieltz D, Hassan A, Yates JR, 3rd, Berger SL, Workman JL. 1999. The ADA complex is a distinct histone acetyltransferase complex in Saccharomyces cerevisiae. Mol Cell Biol 19:6621-31.

19. Sendra R, Tse C, Hansen JC. 2000. The yeast histone acetyltransferase A2 complex, but not free Gcn5p, binds stably to nucleosomal arrays. J Biol Chem 275:24928-34.

20. Sellam A, Askew C, Epp E, Lavoie H, Whiteway M, Nantel A. 2009. Genome-wide mapping of the coactivator Ada2p yields insight into the functional roles of SAGA/ADA complex in Candida albicans. Mol Biol Cell 20:2389-400.

21. Shivarathri R, Tscherner M, Zwolanek F, Singh NK, Chauhan N, Kuchler K. 2019. The fungal histone acetyl transferase Gcn5 controls virulence of the human pathogen Candida albicans through multiple pathways. Sci Rep 9:9445.

22. Kounatidis I, Ames L, Mistry R, Ho HL, Haynes K, Ligoxygakis P. 2018. A hostpathogen interaction screen Iidentifies ada2 as a mediator of Candida glabrata defenses against reactive oxygen species. G3 (Bethesda) 8:1637-1647.

23. Yu SJ, Chang YL, Chen YL. 2018. Deletion of ADA2 increases antifungal drug susceptibility and virulence in Candida glabrata. Antimicrob Agents Chemother 62.

24. Schwarzmuller T, Ma B, Hiller E, Istel F, Tscherner M, Brunke S, Ames L, Firon A, Green B, Cabral V, Marcet-Houben M, Jacobsen ID, Quintin J, Seider K, Frohner I, Glaser W, Jungwirth H, Bachellier-Bassi S, Chauvel M, Zeidler U, Ferrandon D, Gabaldon T, Hube B, d'Enfert C, Rupp S, Cormack B, Haynes K, Kuchler K. 2014. Systematic phenotyping of a large-scale Candida glabrata deletion collection reveals novel antifungal tolerance genes. PLoS Pathog 10.

25. Deckert J, Struhl K. 2002. Targeted recruitment of Rpd3 histone deacetylase represses transcription by inhibiting recruitment of Swi/Snf, SAGA, and TATA binding protein. Mol Cell Biol 22:6458-70.

26. Thornton JL, Westfield GH, Takahashi YH, Cook M, Gao X, Woodfin AR, Lee JS, Morgan MA, Jackson J, Smith ER, Couture JF, Skiniotis G, Shilatifard A. 2014. Context dependency of Set1/COMPASS-mediated histone H3 Lys4 trimethylation. Genes Dev 28:115-20.

27. Raman SB, Nguyen MH, Zhang Z, Cheng S, Jia HY, Weisner N, Iczkowski K, Clancy CJ. 2006. Candida albicans SET1 encodes a histone 3 lysine 4 methyltransferase that contributes to the pathogenesis of invasive candidiasis. Mol Microbiol 60:697-709. 
28. Bruno VM, Kalachikov S, Subaran R, Nobile CJ, Kyratsous C, Mitchell AP. 2006. Control of the $\mathrm{C}$. albicans cell wall damage response by transcriptional regulator Cas5. PLoS Pathog 2:e21.

29. Zordan RE, Ren Y, Pan SJ, Rotondo G, De Las Penas A, Iluore J, Cormack BP. 2013. Expression plasmids for use in Candida glabrata. G3 3:1675-86.

30. Yeaman MR, Yount NY, Waring AJ, Gank KD, Kupferwasser D, Wiese R, Bayer AS, Welch WH. 2007. Modular determinants of antimicrobial activity in platelet factor-4 family kinocidins. Biochim Biophys Acta 1768:609-19.

31. Brennan M, Thomas DY, Whiteway M, Kavanagh K. 2002. Correlation between virulence of Candida albicans mutants in mice and Galleria mellonella larvae. FEMS Immunol Med Microbiol 34:153-7.

32. Wiederhold NP, Najvar LK, Bocanegra R, Molina D, Olivo M, Graybill JR. 2007. In vivo efficacy of anidulafungin and caspofungin against Candida glabrata and association with in vitro potency in the presence of sera. Antimicrob Agents Chemother 51:16161620 . 
Table 1. Results of the mutant screen. List of genes that were required for resistance to both protamine and caspofungin.

\begin{tabular}{|c|c|c|}
\hline Systematic Name & $\begin{array}{l}\text { Gene } \\
\text { Name }\end{array}$ & Putative Function \\
\hline CAGLOK06193g & $A D A 2$ & $\begin{array}{l}\text { Transcriptional coactivator; component of the Spt-Ada-Gcn5 } \\
\text { acetyltransferase (SAGA) complex }\end{array}$ \\
\hline CAGLOF08283g & GCN5 & $\begin{array}{l}\text { Histone acetyltransferase; component of the Spt-Ada-Gcn5 } \\
\text { acetyltransferase (SAGA) complex }\end{array}$ \\
\hline CAGLOF01837g & SPT8 & $\begin{array}{l}\text { Component of the Spt-Ada-Gen5 acetyltransferase (SAGA) } \\
\text { complex }\end{array}$ \\
\hline CAGL0A03322g & HOS2 & $\begin{array}{l}\text { NAD-dependent histone deacetylase; subunit of the Set3 and } \\
\text { Rpd3L complex }\end{array}$ \\
\hline CAGL0B01441g & $R P D 3$ & $\begin{array}{l}\text { Histone deacetylase; regulates transcription and silencing; } \\
\text { subunit of the Rpd3L complex }\end{array}$ \\
\hline CAGLOM02585g & SPP1 & $\begin{array}{l}\text { Transcriptional activator; histone methyltransferase; part of } \\
\text { Set1/COMPASS complex }\end{array}$ \\
\hline CAGLOM06831g & $C R Z 1$ & $\begin{array}{l}\text { Transcription factor; downstream component of the } \\
\text { calcineurin signaling pathway }\end{array}$ \\
\hline CAGL0J10274g & MKS1 & Pleiotropic negative transcriptional regulator \\
\hline
\end{tabular}




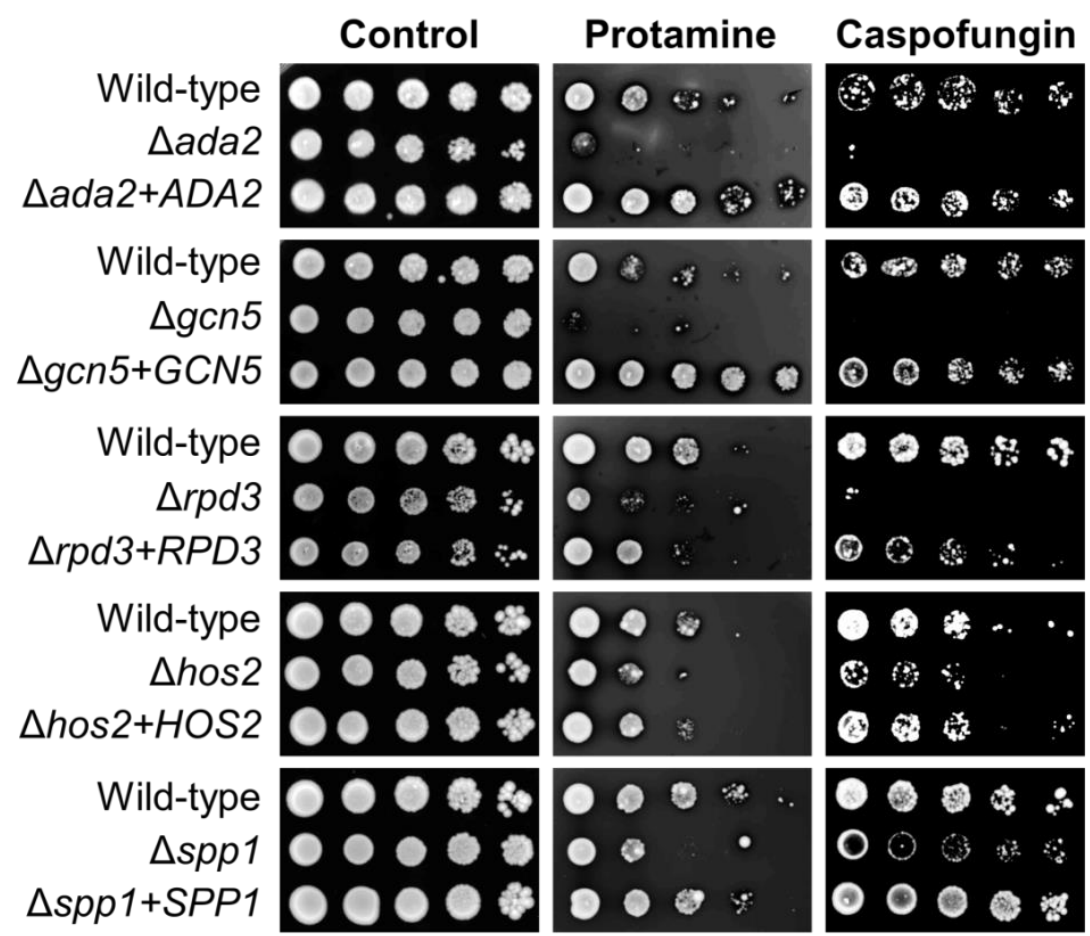

Fig. 1. Susceptibility of the various $C$. glabrata mutants to protamine and caspofungin. Serial 10-fold dilutions of the indicated strains were grown on YPD agar alone or on agar containing either protamine or caspofungin at $37^{\circ} \mathrm{C}$ and then imaged after $48 \mathrm{~h}$. 


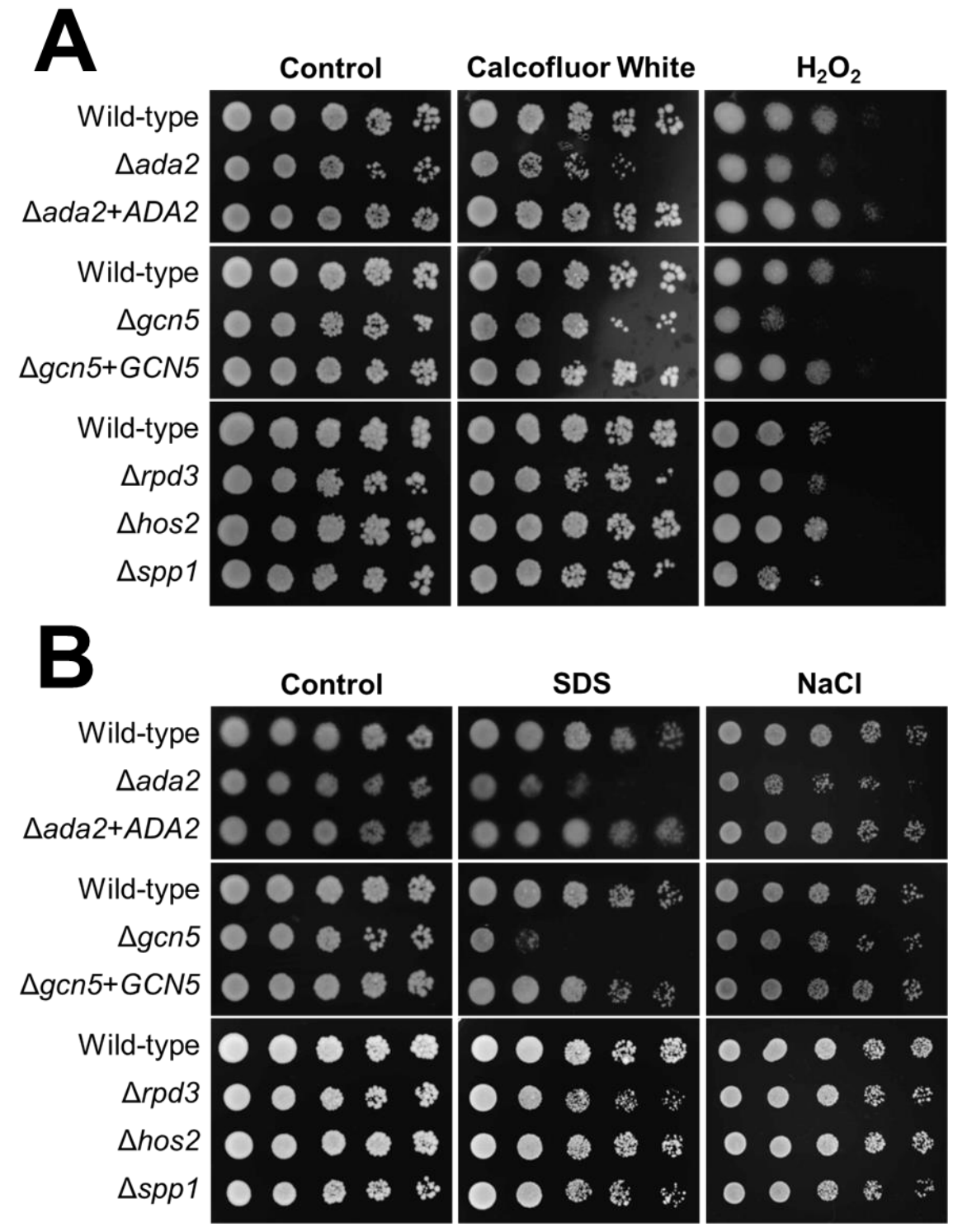

Fig. 2. Susceptibility of $\boldsymbol{C}$. glabrata mutants to additional stressors. Serial 10 -fold dilutions of the indicated strains were grown on YPD agar alone or on agar containing the indicated stressors at $37^{\circ} \mathrm{C}$ and then imaged after $48 \mathrm{~h}$. 

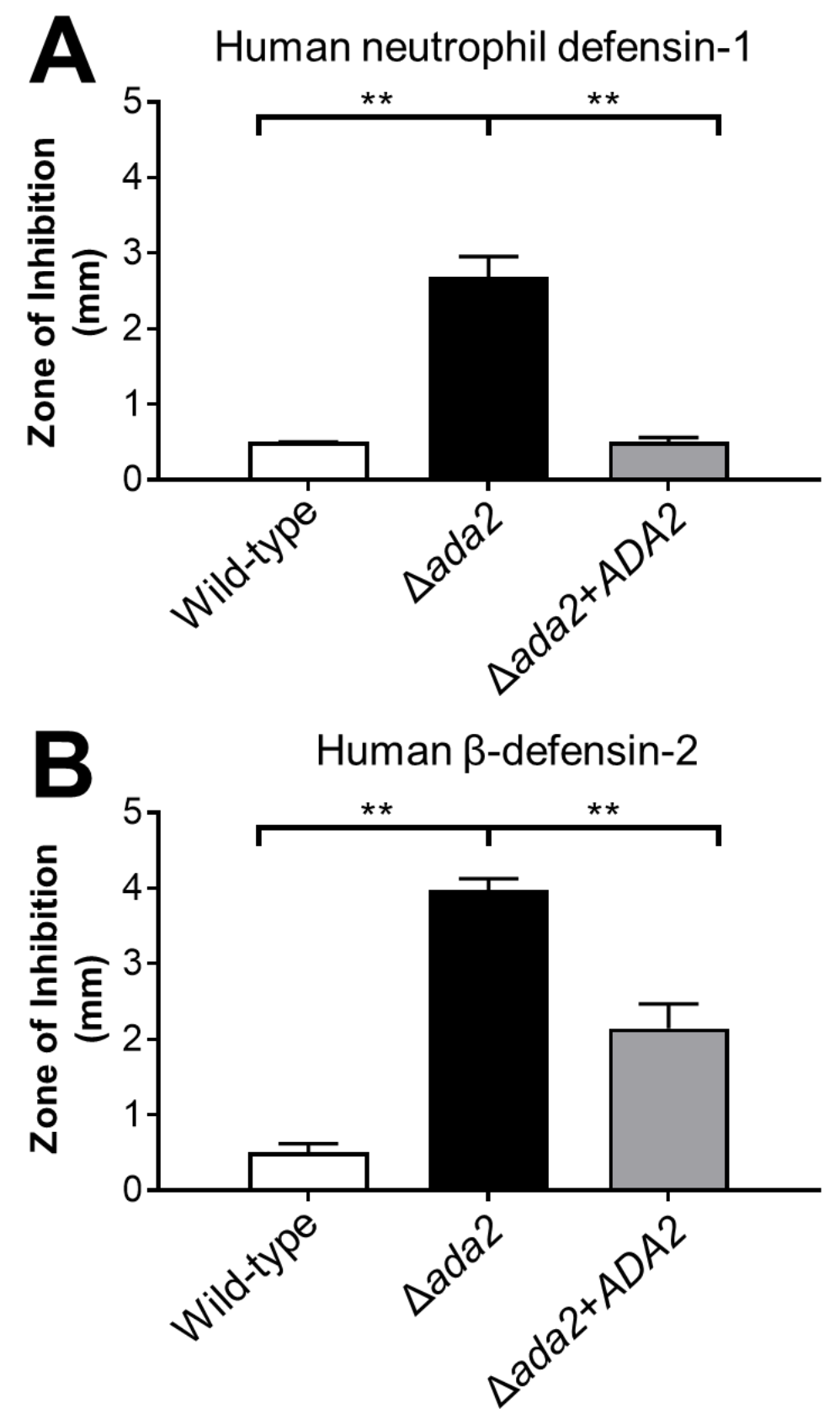

Fig. 3. The ada2 $\Delta$ mutant has increased susceptibility to human defensins. The susceptibility of the indicated strains to human neutrophil defensin-1 (hNP-1) (A) and human $\beta$ defensin-2 (hBD-2) (B) was determined by radial diffusion assay. Results are the mean \pm SD duplicate determinations. ${ }^{*} P<0.01$ by analysis of variance with the Tukey test for multiple comparisons. 

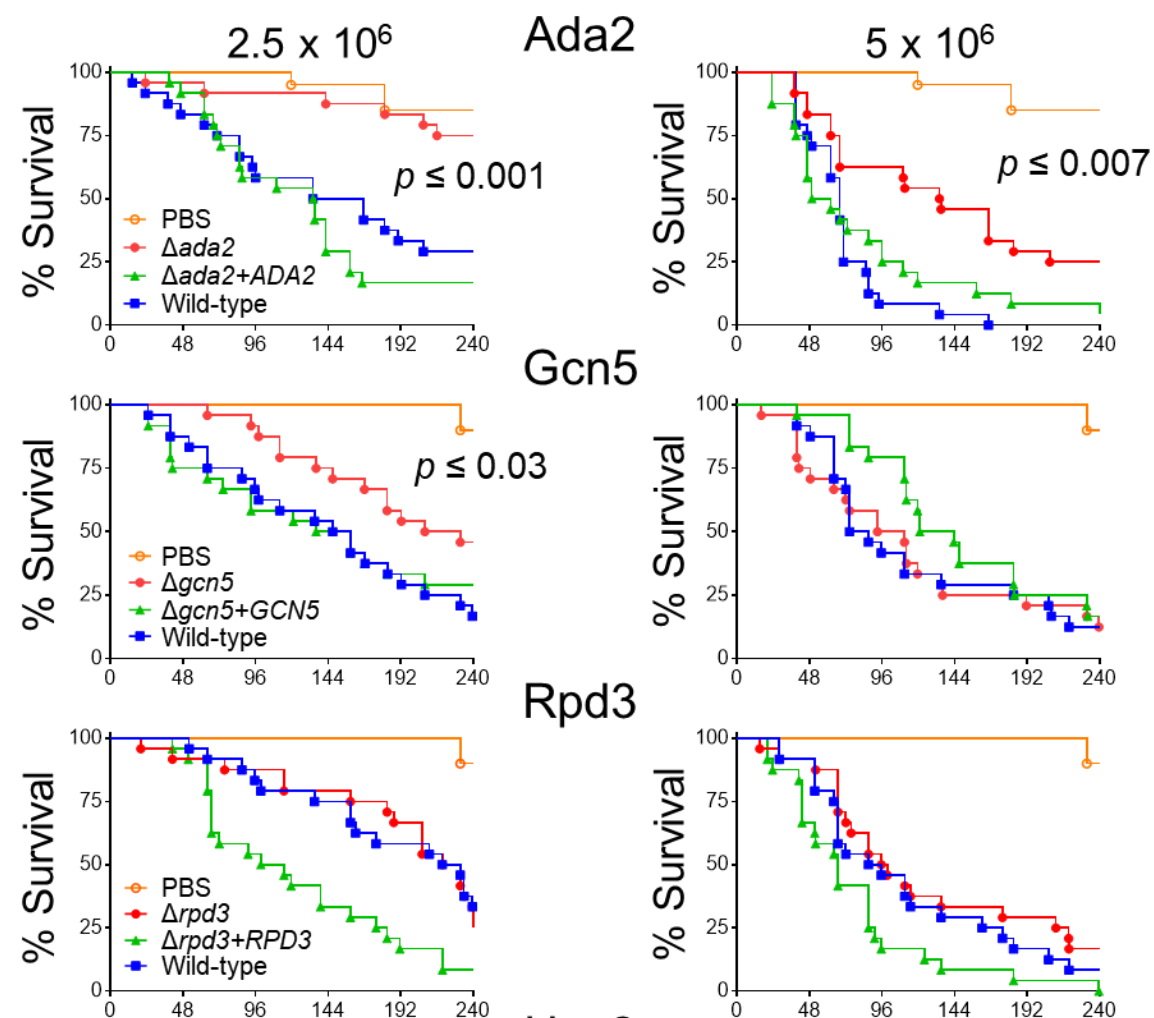

Rpd3
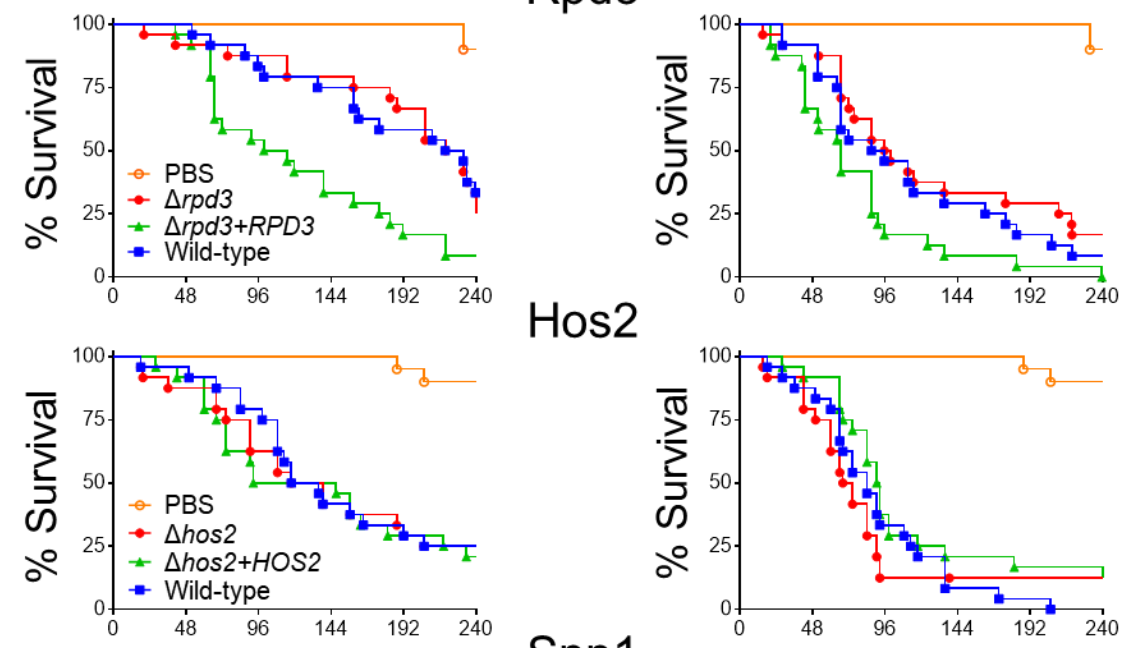

Hos2
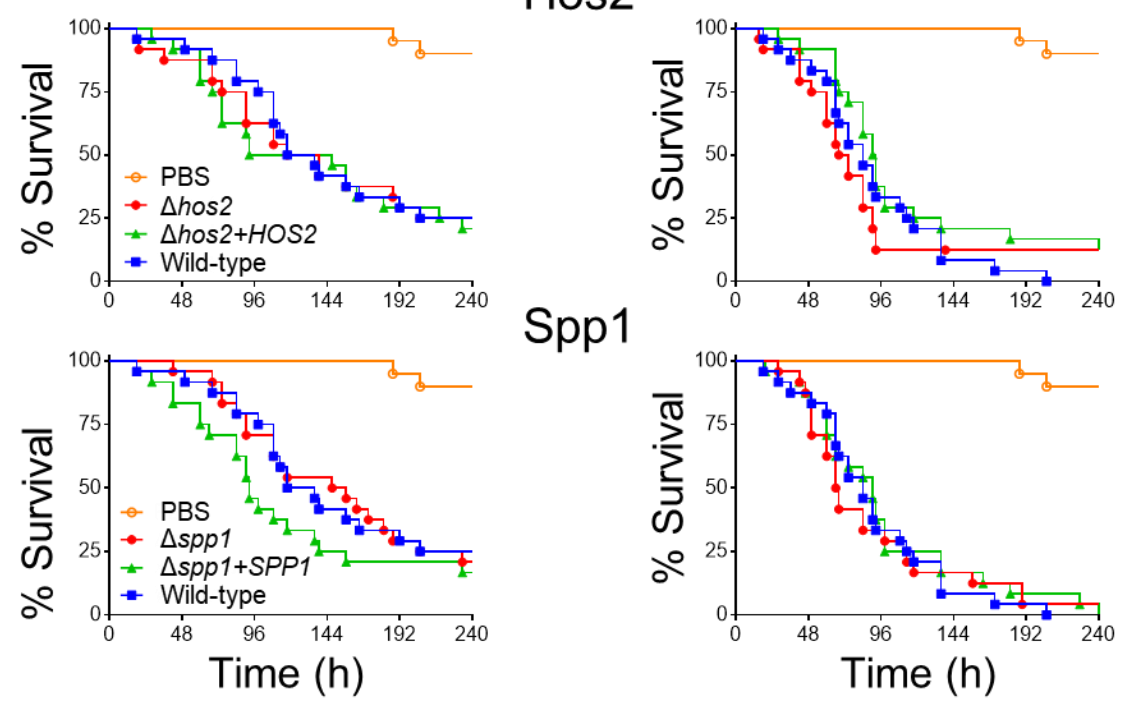

Fig. 4. Virulence testing in G. mellonella. G. mellonella larvae were infected with either $2.5 \mathrm{x}$ $10^{6}$ cells (left panels) or $5 \times 10^{6}$ (right panel) cells of the indicated strains of C. glabrata and then monitored twice daily for survival. Survival curves are the combined results of 2 independent experiments for a total of 24 larvae per strain. The indicated $p$ values were determined using the Gehan-Breslow-Wilcoxon test, comparing the larvae infected with the deletion mutant with those infected with the wild-type and complemented strains. 


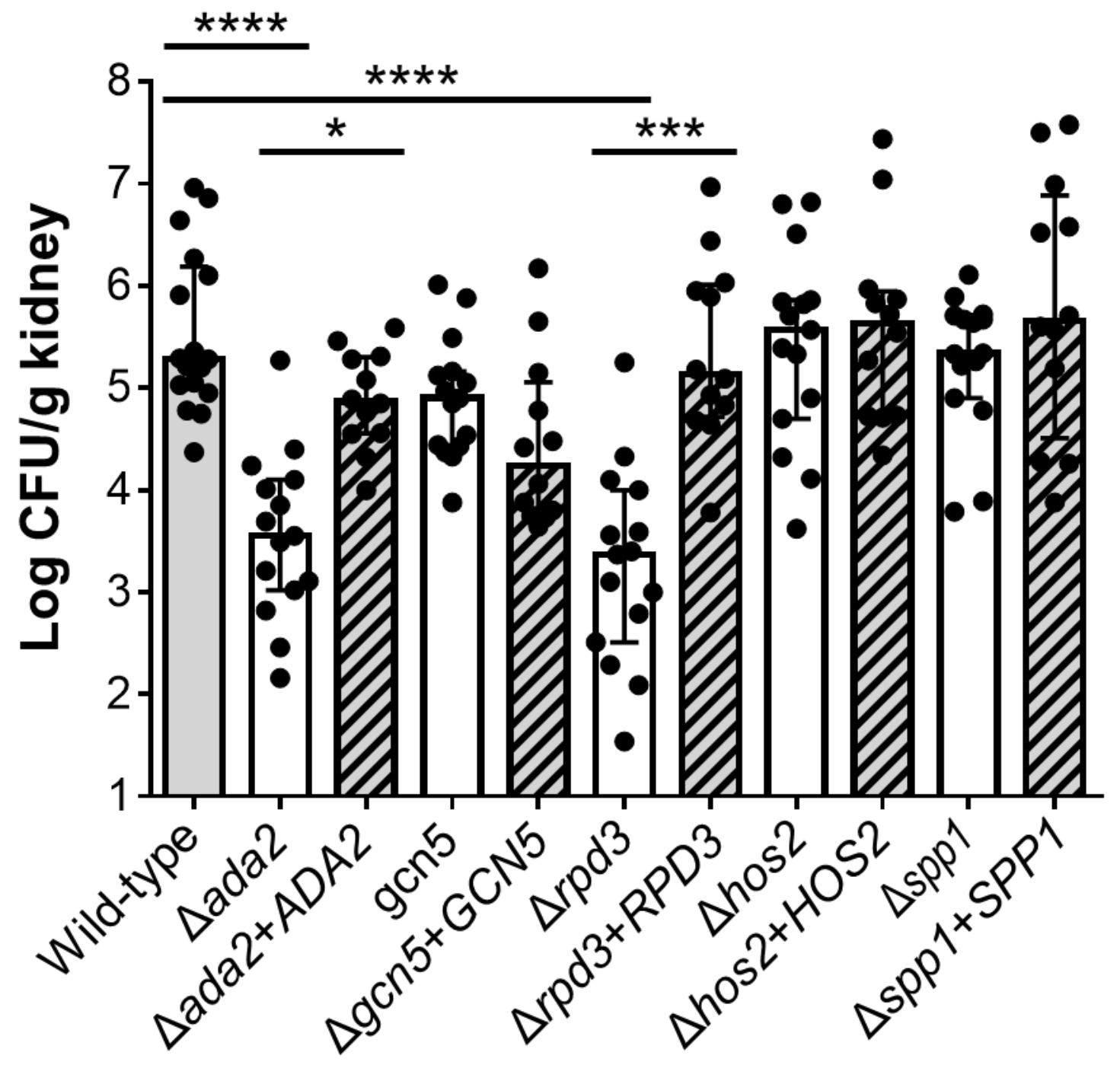

Fig. 5. Virulence testing in mice. Neutropenic mice were inoculated intravenously with the indicated strains of $C$. glabrata. After 8 days, the mice were sacrificed and the kidney fungal burden was determined. Results are the median \pm interquartile range of the combined results of two independent experiments for a total of $12-17$ mice per strain. $* P<0.05$, $* * * P<0.001$, $* * * * P<0.0001$ by the Kruskal-Wallis test for multiple comparisons. 
Table S1. Results of screening the deletion library for susceptibility to protamine and caspofungin. 